\title{
EFFECT OF PULMONARY HYPERTENSION ON INTRADIALYTIC HYPO- TENSION AMONG PATIENTS WITH END STAGE RENAL DISEASE
}

\author{
Sukrisd Koowattanatiancha*, Akaphol Kaladee**, Patchara Kochaiyapatana*, Thammaporn \\ Kajornsin*, Raweewan Witoon***
}
*Division of Cardiology, Department of Medicine, Burapha Hospital, Burapha University, Chonburi, Thailand
** School of Health Science, Sukhothai Thammathirat Open University, Nonthaburi, Thailand *** Division of Nephrology, Department of Medicine, Burapha Hospital, Burapha University, Chonburi, Thailand

\begin{abstract}
Background: Intradialytic hypotension (IDH) is an important problem in end stage renal disease (ESRD). Therefore, this study aimed to assess the effect of pulmonary hypertension (PHT) on IDH among patients with ESRD using transthoracic echocardiography.

Methods: In this prospective etiognostic study, transthoracic echocardiography was performed among patients with ESRD in Burapha University Hospital, Thailand. The hemodialytic flow chart data of patients in the hemodialysis unit was collected to ascertain whether these patients presented IDH. The baseline clinical hemodialysis profiles and echocardiographic findings were analyzed using univariate predictors of IDH. Multivariate risk regression was used to identify independent predictors of IDH.

Results: A total of 35 patients with ESRD were enrolled between June 2020 and March 2021. Of these, 16 had PHT (45.7\%). The incidence of IDH was 48.5\%. All patients exhibited a normal left ventricular ejection fraction. No significant difference was observed of RVSP between frequent-IDH group and occasional-IDH group $(45.33 \pm 11.62 \mathrm{mmHg}$ and $41.06 \pm 13.78 \mathrm{mmHg}$, respectively, $p=0.401)$. Using univariate analysis, being female, left ventricular mass index, left ventricular ejection fraction and PHT were significantly associated with IDH. No factors were indicated related to IDH occurrence using multivariate analysis. Nevertheless, female patients with ESRD presenting PHT illustrated a tendency to have IDH. This was evidenced by the risk ratio of being female and patients with PHT being 3.13 (95\% CI: 0.74-13.30) and 2.18 (95\% CI: 0.34-7.06), respectively.

Conclusion: Patients with ESRD presenting PHT showed a higher tendency of developing IDH during hemodialysis than patients with ESRD without PHT. The difference however was statistically insignificant.
\end{abstract}

Keywords: End stage renal disease, Intradialytic hypotension, Pulmonary hypertension

J Southeast Asian Med Res 2022; 6: e0098

https://doi.org/10.55374/jseamed.v6i0.98

Correspondence to:

Koowattanatiancha S, Division of Cardiology, Department of Medicine, Burapha Hospital, Burapha University, Chonburi, Thailand

E-mail: sukrisd.ko@buu.ac.th

Received: 4 August 2021

Revised: 29 November 2021

Accepted: 3 December 2021 


\section{Introduction}

Intradialytic hypotension (IDH) is one of the complications associated with dialysis procedures, occurring at about 20 to $48 \%^{(1-3)}$ of patients with end stage renal disease (ESRD). The condition is important in clinical settings because it could lead to even more serious complications such as an increase of mortality rate $^{(4,5)}$ and myocardial ischemia from decreasing coronary blood flow. ${ }^{(6)}$

IDH condition has been hypothesized to cause a lower cardiac output. In fact, both left-sided and right-sided cardiac outputs are closely related. When the right-sided cardiac output is lower, whatever the cause, low blood pressure will occur. Therefore, hemodialytic sessions involving a removal of body fluid from blood vessels, resulting in a decreasing preload in the right ventricle, should be particularly cautioned because it may reduce the right-sided cardiac output. Special attention should be paid to the occurrence of associated conditions such as pulmonary hypertension (PHT).

Approximately 26 to $66 \%$ of patients with $\operatorname{ESRD(7-11)~already~being~treated~by~hemodialysis~}$ present PHT and are correlated with cardiovascular deaths. ${ }^{(9)}$ Also, PHT is a strong independent predictor of mortality among patients undergoing hemodialysis. ${ }^{(12)}$

No related studies have significantly documented the relationship between PHT and IDH. Therefore, this study aimed to assess the effect of PHT on IDH among patients with ESRD using transthoracic echocardiography.

\section{Methods}

\section{Study design and population}

This constituted a prospective etiognostic study that performed echocardiography among patients with ESRD in Burapha University Hospital, Thailand between June 2020 and December 2020. After that, the hemodialysis flow chart data of patients in the hemodialysis unit was collected to ascertain whether these patients presented IDH. The protocol was approved for ethics considerations by the BUU Ethics Institutional Review Board (HS021/2563). All patients provided written informed consent.
The sample size was calculated by comparing the mean of two independent groups. A related study ${ }^{(13)}$ indicated that on average, patients with ESRD receiving hemodialysis exhibited pulmonary artery systolic pressure about 33.9 $\pm 10.6 \mathrm{mmHg}$. Moreover, experts suggested that patients with ESRD and IDH should have higher pulmonary artery systolic pressure than patients with ESRD without IDH. The difference was expected to be about $10 \mathrm{mmHg}$, which is a value predicting the emergence of poor long term survival. ${ }^{(13)}$ Thus, patients with ESRD and IDH are expected to have pulmonary artery systolic pressure equal to $43.9 \mathrm{mmHg}$. The sample size was calculated to include 48 patients with ESRD in the study (24 patients in each group).

Patients with IDH under the condition that systolic blood pressure decreased by $20 \mathrm{mmHg}$ or more, the mean arterial pressure decreased by more than $10 \mathrm{mmHg}$ or blood pressure was lower than 100/60 with associated symptoms of hypotension including yawning, cramps, nausea, vomiting, tweaking and dizziness. ${ }^{(14)}$ Patients in the frequent-IDH group needed to be particularly treated during the hemodialysis sessions. Examples of treatments included temporarily stopping an ultrafiltration (UF) operation, providing inotropic agents or saline.(15) Patients with frequent-IDH in this study exhibited the aforementioned condition more than three times during a three-month study, whereas patients with occasional-IDH showed the condition less than three times or none during the same timeframe. The hemodialysis chart of patients was reviewed to collect data about 1) general information including age, sex, body mass index (BMI), underlying diseases, causes of chronic renal failure, medicines used, and laboratory results and 2) cardiovascular disease-related information including previous HF hospitalization, coronary artery disease, history of pulmonary hypertension and history of syncope and 3) kidney dialysis related information including weekly frequency of kidney dialysis, length of time of each kidney dialysis, mean net UF weekly, position of catheter, pre/post HD weight, and pre/post blood pressure and heart rate. The inclusion criteria included patients (1) aged more than 18 years (2) having 
treatment of hemodialysis for six months or more, (3) receiving hemodialysis at least twice weekly and at least four hours each session, (4) without history of catheter related infection and (5) without history of abnormal bleeding in the last month. The exclusion criteria included (1) BMI of $40 \mathrm{~kg} / \mathrm{m} 2$ or more, (2) catheter-related infection in the last month, (3) bleeding disorder in the last month, (4) inadequate hemodialysis defined by losing HD twice in the last month, (5) significant left sided valvular heart disease (any aortic/ mitral stenosis), (6) HIV positive status and (7) pregnancy.

\section{Hemodialysis}

All patients with ESRD received dialysis two to three times weekly for four hours each session.

\section{Transthoracic echocardiography}

Echocardiography was performed before hemodialysis to collect important parameters including left ventricular ejection fraction (LVEF), left ventricular mass index (LVMi), pattern of left ventricular geometry, LA volume index, diastolic function, E/e', right atrial pressure, tricuspid regurgitation velocity, right ventricular systolic pressure (RVSP), mean pulmonary arterial pressure (mean PAP) using Abbas's formula and right ventricular systolic function (tricuspid annular plane systolic excursion and peak velocity of tricuspid annulus). Echocardiogram was performed by a cardiologist in Burapha Hospital. We used the American Society of Echocardiography guidelines and recommendations to assess and measure these parameters. The cardiologist performed all examinations using a diagnostic ultrasound system (Philips EPIQ CVx with a Philips X5-1 MHz Phased Array Probe).

\section{Statistical Analysis}

Subject characteristics were described using descriptive statistics, including frequency and percentage for categorical variables. Continuous variables were reported as means, standard deviation of normally distributed variables and median, minimum and maximum of normally distributed variables. Factors to predict IDH used the independent t-test for continuous variables and categorical variables used the Fisher exact test. Variables found to be significant in the univariate analysis, were entered in a multivariate risk regression analysis (backward elimination). For all tests performed, a two-tailed $p<0.05$ was considered statistically significant.

\section{Results}

The data of 40 patients with ESRD were collected. Three patients were excluded from the study because parameter values using an echocardiogram were unable to be collected. Two patients with severe valvular heart disease were excluded. Finally, a total of 35 patients with ESRD were analyzed. Of these, 16 had PHT (45.7\%), and incidence of IDH was $48.6 \%$. General information of patients in both groups are shown in Table $\mathbf{1}$ and hemodialysis profiles are shown in Table 2.

All patients exhibited normal LVEF and right ventricular systolic function (tricuspid annular plane systolic excursion and peak velocity of tricuspid annulus). Most patients presented Grade I diastolic function and concentric hypertrophy of the left ventricular geometric pattern. The echocardiographic data are shown in Table 3.

The primary research outcome was that a relationship between PHT and IDH was insignificant. When the dataset was subjected to univariate analysis, variables with a significant correlation with IDH were being female, LVMi, LVEF and PHT. The RVSP of the frequent-IDH group and the occasional-IDH group appeared to be $45.33 \pm 11.6224 .86 \pm 8.16 \mathrm{mmHg}$, respectively $(p=0.234)$. 
Table 1. Clinical characteristics of frequent-IDH and occasional-IDH groups

\begin{tabular}{|c|c|c|c|}
\hline \multirow{2}{*}{ Characteristic } & \multirow{2}{*}{$\frac{\text { Frequent-IDH }}{(\mathrm{n}=17)}$} & \multirow{2}{*}{$\frac{\text { Occasional-IDH }}{(n=18)}$} & \multirow{2}{*}{$p$-value } \\
\hline & & & \\
\hline Female, n (\%) & $14(73.7)$ & $5(26.3)$ & 0.002 \\
\hline Age (years, mean \pm sd) & $68.82 \pm 12.51$ & $66.11 \pm 16.35$ & 0.567 \\
\hline Body weight $(\mathrm{kg}$, mean $\pm \mathrm{sd})$ & $59.18 \pm 16.14$ & $60.78 \pm 12.94$ & 0.747 \\
\hline Body mass index $\left(\mathrm{kg} / \mathrm{m}^{2}\right.$, mean $\left.\pm \mathrm{sd}\right)$ & $24.32 \pm 5.8$ & $23.61 \pm 4.74$ & 0.691 \\
\hline \multicolumn{4}{|l|}{ Underlying disease, n (\%) } \\
\hline Hypertension & $11(45.8)$ & $13(54.2)$ & 0.725 \\
\hline Coronary artery disease & $2(28.6)$ & $5(71.4)$ & 0.402 \\
\hline Stroke & $2(66.7)$ & $1(33.3)$ & 0.603 \\
\hline Diabetes mellitus & $4(43.8)$ & $9(56.2)$ & 0.738 \\
\hline Dyslipidemia & $15(60.0)$ & $10(40.0)$ & 0.06 \\
\hline Previous heart failure & $1(20.0)$ & $4(80.0)$ & 0.338 \\
\hline Etiology of ESRD, n (\%) & & & 0.49 \\
\hline Diabetes mellitus & $8(44.4)$ & $10(55.6)$ & \\
\hline HTN (HT nephrosclerosis) & $5(62.5)$ & $3(37.5)$ & \\
\hline Glomerulonephritis & 0 & $1(100.0)$ & \\
\hline Unknown & $4(50.0)$ & $4(50.0)$ & \\
\hline $\begin{array}{l}\text { Number of Anti HTN drugs } \\
(\text { mean } \pm \text { SD) }\end{array}$ & $1.88 \pm 1.22$ & $2.23 \pm 1.31$ & 0.433 \\
\hline Nitrate, $\mathrm{n}(\%)$ & 0 & $2(100.0)$ & 0.486 \\
\hline Beta blocker, n (\%) & $4(33.3)$ & $8(66.7)$ & 0.289 \\
\hline CCB, n $(\%)$ & $10(47.6)$ & $11(52.4)$ & 0.582 \\
\hline ACEI, n $(\%)$ & $1(50.0)$ & $1(50.0)$ & 0.743 \\
\hline $\mathrm{ARB}, \mathrm{n}(\%)$ & $5(50.0)$ & $5(50.0)$ & 0.604 \\
\hline Diuretic, n (\%) & $11(47.8)$ & $12(52.2)$ & 0.592 \\
\hline Hydralazine, n (\%) & $1(25.0)$ & $3(75.0)$ & 0.603 \\
\hline \multicolumn{4}{|l|}{ Laboratory investigation } \\
\hline $\mathrm{Hb}(\mathrm{mg} / \mathrm{dL}$, mean $\pm \mathrm{SD})$ & $9.45 \pm 1.54$ & $9.76 \pm 1.39$ & 0.545 \\
\hline Albumin (mg/dL, mean \pm SD) & $3.8 \pm 0.36$ & $3.77 \pm 0.31$ & 0.77 \\
\hline Total calcium $(\mathrm{mg} / \mathrm{dL}$, mean $\pm \mathrm{SD})$ & $8.85 \pm 0.98$ & $8.92 \pm 0.8$ & 0.813 \\
\hline Phosphate (mg/dL, mean \pm SD) & $4.68 \pm 1.51$ & $4.32 \pm 0.93$ & 0.415 \\
\hline PTH level (pg/mL, mean \pm SD) & $262.11 \pm 242.11$ & $284.73 \pm 287.04$ & 0.811 \\
\hline
\end{tabular}

Values presented as mean $\pm \mathrm{SD}$ or $\mathrm{n}(\%), p$-values corresponded to independent- $\mathrm{t}$ test and Fisher's exact test. ESRD: end stage renal disease, anti HTN drugs: antihypertensive drugs, ACEI: angiotensin-converting enzyme inhibitors, ARB: angiotensin II receptor blockers, CCB: calcium channel blockers, Hb: hemoglobin, PTH: parathyroid hormone 
Table 2. Hemodialysis profiles of frequent-IDH and occasional-IDH groups

\begin{tabular}{|c|c|c|c|}
\hline \multirow{2}{*}{ Hemodialysis profile } & Frequent-IDH group & Occasional-IDH group & \multirow{2}{*}{$p$-value } \\
\hline & $(n=17)$ & $(\mathrm{n}=18)$ & \\
\hline IDH symptoms, n (\%) & & & 0.198 \\
\hline None & $7(31.8)$ & $15(68.2)$ & \\
\hline Fainting & $4(66.6)$ & $2(34.4)$ & \\
\hline Dyspnea & $1(100.0)$ & 0 & \\
\hline chest pain & $1(100.0)$ & 0 & \\
\hline abdominal pain & $1(100.0)$ & 0 & \\
\hline muscle cramping & $2(66.7)$ & $1(33.3)$ & \\
\hline back pain & $1(100.0)$ & 0 & \\
\hline Frequency of dialysis, $\mathrm{n}(\%)$ & & & 0.315 \\
\hline 2 sessions/week & $9(42.9)$ & $12(57.1)$ & \\
\hline 3 sessions/week & $8(57.1)$ & $6(42.9)$ & \\
\hline Mean Net UF, (ml, mean \pm sd) & $2535.29 \pm 829.11$ & $2742.22 \pm 1283.69$ & 0.577 \\
\hline $\mathrm{BFR},(\mathrm{ml} / \mathrm{min}$, mean $\pm \mathrm{sd})$ & $329.41 \pm 25.36$ & $327.78 \pm 30.78$ & 0.865 \\
\hline Dialysis membrane & & & 0.591 \\
\hline HDF 80 & $9(60.0)$ & $6(40.0)$ & \\
\hline HDF 100 & $2(25.0)$ & $6(75.0)$ & \\
\hline Elisio 170 & $3(42.9)$ & $4(57.1)$ & \\
\hline Elisio 190 & $1(50.0)$ & $1(50.0)$ & \\
\hline Elisio 210 & $2(66.7)$ & $1(33.3)$ & \\
\hline PreHD SBP, $(\mathrm{mmHg}$, mean $\pm \mathrm{SD})$ & $141.06 \pm 27.88$ & $131.28 \pm 24.48$ & 0.274 \\
\hline PreHD DBP, $(\mathrm{mmHg}$, mean \pm SD) & $64.24 \pm 17.49$ & $61.89 \pm 15.13$ & 0.673 \\
\hline PreHD HR, (bpm mean \pm SD) & $76.76 \pm 15.83$ & $78.06 \pm 17.13$ & 0.819 \\
\hline Position of catheters, n (\%) & & & 0.67 \\
\hline AVF & $10(47.6)$ & $11(52.4)$ & \\
\hline AVG & $1(33.3)$ & $2(66.7)$ & \\
\hline Perm cath & $4(44.4)$ & $5(55.6)$ & \\
\hline DLC & $2(100.0)$ & 0 & \\
\hline
\end{tabular}

Values presented as mean $\pm \mathrm{SD}$ or $\mathrm{n}(\%), p$-values corresponded to independent-t test and Fisher's exact test.

Mean net UF: mean net ultrafiltration, BFR: blood flow rate, PreHD SBP: prehemodialysis systolic blood pressure, PreHD DBP: prehemodialyisis diastolic blood pressure, PreHD HR: prehemodialysis heart rate, AVF: arteriovenous fistula, AVG: arteriovenous graft, DLC: double lumen catheter.

These variables and other variables that were likely to be related to the occurrence of IDH including diabetes, coronary artery disease, albumin level, presystolic blood pressure, prediastolic blood pressure, LVMi and LVEF were then analyzed using multivariable analysis. Results showed that no factors were related to IDH occurrence. Nevertheless, female patients and patients with PHT illustrated a tendency to have IDH. This was evidenced by the risk ratio of being female and patients with PHT at $3.13(95 \%$ $\mathrm{CI}=0.74-13.30)$ and $2.18(95 \% \mathrm{CI}=0.34-7.06)$, respectively. multivariate risk regression analysis to determine factors associated with IDH is shown in Table 4. 
Table 3. Echocardiographic data between frequent-IDH and occasional-IDH groups

\begin{tabular}{|c|c|c|c|}
\hline \multirow{2}{*}{ Echocardiographic findings } & $\begin{array}{c}\text { Frequent-IDH } \\
\text { group }(n=17)\end{array}$ & $\begin{array}{l}\text { Occasional-IDH } \\
\text { group }(\mathrm{n}=18)\end{array}$ & \multirow[t]{2}{*}{$p$-value } \\
\hline & $($ mean $\pm \mathrm{SD})$ & $($ mean \pm SD $)$ & \\
\hline LVDd (mm) & $44.07 \pm 5.8$ & $41.47 \pm 5.77$ & 0.193 \\
\hline LV mass index $\left(\mathrm{g} / \mathrm{m}^{2}\right)$ & $128.48 \pm 26.52$ & $110.22 \pm 21.69$ & 0.032 \\
\hline RWT & $0.62 \pm 0.14$ & $0.69 \pm 0.14$ & 0.173 \\
\hline LV geometry, n (\%) & & & 0.125 \\
\hline concentric remodeling & $2(22.2)$ & $7(77.8)$ & \\
\hline concentric hypertrophy & $14(56.0)$ & $11(44.0)$ & \\
\hline LVEF $(\%)$ & $64.08 \pm 11.19$ & $72.87 \pm 5.89$ & 0.006 \\
\hline Diastolic function, $\mathrm{n}(\%)$ & & & 0.429 \\
\hline normal & $1(25.0)$ & $3(75.0)$ & \\
\hline grade I & $13(54.8)$ & $11(45.8)$ & \\
\hline grade II & $2(33.3)$ & $4(66.7)$ & \\
\hline grade III & $1(100.0)$ & 0 & \\
\hline $\mathrm{E} / \mathrm{e}$ & $17.83 \pm 7.01$ & $15.15 \pm 6.32$ & 0.243 \\
\hline LA volume index (mL/sqm) & $47.38 \pm 13.85$ & $50.12 \pm 14.92$ & 0.579 \\
\hline TAPSE (mm) & $25.42 \pm 3.86$ & $25.29 \pm 4.78$ & 0.931 \\
\hline $\begin{array}{l}\text { Peak velocity of tricuspid annulus } \\
(\mathrm{cm} / \mathrm{sec})\end{array}$ & $12.7 \pm 2.51$ & $11.99 \pm 1.62$ & 0.326 \\
\hline mean PAP $(\mathrm{mmHg})$ & $28.28 \pm 6.94$ & $24.86 \pm 8.16$ & 0.234 \\
\hline RVSP (mmHg) & $45.33 \pm 11.62$ & $41.06 \pm 13.78$ & 0.401 \\
\hline PHT, n (\%) & $11(68.8)$ & $5(31.3)$ & 0.044 \\
\hline RAP (mmHg) & $8.54 \pm 1.82$ & $8.71 \pm 2.12$ & 0.788 \\
\hline $\mathrm{CO}(\mathrm{mL} / \mathrm{min})$ & $5.31 \pm 2.25$ & $4.32 \pm 0.98$ & 0.100 \\
\hline
\end{tabular}

Values presented as mean $\pm \mathrm{SD}$ or n (\%), $p$-values corresponded to independent-t test and Fisher's exact test.

LVDd: left ventricular dimension in diastole, LV mass index: left ventricular mass index, RWT: relative wall thickness, LV geometry: left ventricular geometry, LVEF: left ventricular ejection fraction, LA volume index: left atrial volume index, TAPSE: tricuspid annular plane systolic excursion, Mean PAP: mean pulmonary arterial pressure RVSP: right ventricular systolic pressure, PHT: pulmonary hypertension, RAP: right atrial pressure, CO: cardiac output.

\section{Discussion}

IDH is a serious and frequent complication of chronic hemodialysis. IDH is the end result of the interaction between ultrafiltration rates, cardiac output and arteriolar tone. ${ }^{(16)}$ As mentioned previously, we hypothesized that the occurrence of IDH among patients undergoing chronic hemodialysis was related to reduced cardiac output and PHT. When developing IDH, the human body exhibits the following adapted mechanisms. ${ }^{(17)}$ First, interstitial fluid moves to blood vessels to refill the blood volume. Second, increased sympathetic outflow to arteriolar vasoconstriction and increased peripheral vascular resistance, helps to maintain BP. Finally, the heart contracts more, causing the rising heart rate to increase cardiac output and raise BP. When all of these mechanisms fail, IDH eventually strikes. Decreased cardiac output plays a key role in the pathophysiology of IDH. Cardiac output depends on preload, afterload, heart rate and contractility. Changes in preload, determined mainly by 
Table 4. Multivariate regression analysis to determine factors associated with intradialytic hypotension

\begin{tabular}{llllll}
\hline Prognostic factor & \multicolumn{1}{c}{$\begin{array}{c}\text { Frequent-IDH } \\
(\mathrm{n}=17)\end{array}$} & $\begin{array}{c}\text { Occasional- } \\
\text { IDH } \\
(\mathrm{n}=18)\end{array}$ & $\begin{array}{c}\text { Risk } \\
\text { Ratio }\end{array}$ & $\begin{array}{c}\text { Adjusted Risk } \\
\text { Ratio (95\% CI) }\end{array}$ & $p$-value \\
\hline $\begin{array}{l}\text { PHT, n (\%) } \\
\text { Sex, n (\%) }\end{array}$ & $11(68.8)$ & $5(31.3)$ & 2.18 & $1.56(0.34-7.06)$ & 0.565 \\
$\quad$ male & $4(23.5)$ & $13(76.5)$ & 1 & & 0.120 \\
$\quad$ female & $14(73.7)$ & $5(26.3)$ & 3.13 & $3.14(0.74-13.30)$ & \\
DM, n (\%) & $8(47.1)$ & $9(52.9)$ & 0.89 & $0.49(0.13-1.92)$ & 0.308 \\
CAD, n (\%) & $3(37.5)$ & $5(62.5)$ & 0.70 & $0.81(0.16-4.18)$ & 0.799 \\
Albumin, (mean \pm SD) & $3.85 \pm 0.41$ & $3.77 \pm 0.31$ & 1.39 & $0.53(0.06-4.30)$ & 0.528 \\
PreSBP (mean \pm SD) & $75.0 \pm 21.97$ & $114.5 \pm 20.63$ & 1.0 & $0.98(0.94-1.04)$ & 0.666 \\
PreDBP (mean \pm SD) & $42.17 \pm 13.36$ & $59.67 \pm 13.91$ & 1.0 & $0.98(0.94-1.04)$ & 0.538 \\
LVMi, (mean \pm SD) & $128.48 \pm 26.52$ & $110.22 \pm 21.69$ & 1.01 & $1.0(0.97-1.03)$ & 0.798 \\
LVEF, (mean \pm SD) & $64.08 \pm 11.19$ & $72.87 \pm 5.89$ & 0.96 & $0.97(0.91-1.04)$ & 0.427 \\
\hline
\end{tabular}

PHT: pulmonary hypertension, DM: diabetes mellitus, CAD: coronary artery disease, PreSBP: presystolic blood pressure, PreDBP: prediastolic blood pressure, LVMi: left ventricular mass index, LVEF: left ventricular ejection fraction

intravascular volume, seem to play a major role in the development of IDH. ${ }^{(16)}$ Therefore during our hemodialysis sessions, intravascular fluid has to be removed. Patients presenting pulmonary hypertension may experience induced IDH due to decreasing cardiac output.

multivariate risk regression revealed that such a relationship does not exist. However, the chance that patients with ESRD and PHT condition (indicated by their RVSP being equal or greater than $>50 \mathrm{mmHg}$ or their mean PAP by Abbas's formula being equal or greater than $>25 \mathrm{mmHg}$ ) will develop IDH 2.18 times that of patients without PHT. The pathogenesis of PHT in this population remains poorly understood. Reported associations include arteriovenous fistulae, cardiac dysfunction, fluid overload, bone mineral disorder and non-biocompatible dialysis membranes. ${ }^{(18)}$

This study also illustrated that female patients had a higher chance of developing IDH than male patients. In particular, our multivariate risk regression analysis indicated that the chance of female patients with IDH was 3.13 times than that of male patients. This finding was consistent with several studies. For example, Andras Tisler et al. ${ }^{(19)}$ found that being female was a statistically significant factor of IDH occurrence. Similarly, Johanna et al. ${ }^{(20)}$ examining the prevalence of IDH among patients with conventional hemodialysis, found that four determining factors of IDH included diabetes, high interdialytic weight gain, being female, and low body weight. Studies of Johanna Kuipers et al. ${ }^{(21)}$ and Orofino L et al. ${ }^{(22)}$ also found similar results, that female patients with low body weight increases the chance of having IDH even further. This could be explained in that females in general have lower body weight and, consequently, have a higher UF rate $(\mathrm{mL} / \mathrm{h} / \mathrm{kg}$ body weight) during hemodialysis for a similar interdialytic weight gain than males. ${ }^{(20)}$

Patients with frequent IDH exhibit higher LVMi than patients with occasional IDH indicating that these patients have left ventricular hypertrophy (LVH). For patients with chronic renal failure, the prevalence of $\mathrm{LVH}$ increases progressively as renal function deteriorates. The development of LVH results from coronary hypoperfusion, 
myocardial stunning and renin-angiotensinaldosterone system dysregulation. Finally, LVH actively contributes to IDH occurrence, through the induction of LV stiffening, myocardial ischemia and arrhythmia. ${ }^{(23)}$

Barberato et al. ${ }^{(24)}$ observed that patients experiencing ESRD with LVEF less than 50\% and LA volume index greater than $35 \mathrm{~mL} / \mathrm{m} 2$ indicated diastolic dysfunction, which is a factor that can significantly predict the occurrence of IDH. This study found that both groups of patients (frequent-IDH and occasional-IDH) exhibit normal LVEF, i.e., greater than $60 \%$, and normal cardiac output measured by an echocardiogram. Therefore, high LA volume index will not necessarily cause IDH during chronic hemodialysis.

Apart from patients with ESRD, patients with PHT must be treated with noncardiac operations. This patient group represented an important risk factor for increased perioperative morbidity and mortality. In other words, these patients illustrated a significantly increased risk for hemodynamic instability. ${ }^{(25)}$ Thus, physicians have to give special attention to these patients by monitoring closely, optimizing systemic BP, oxygenating and ventilating, avoiding exacerbating factors, and using vasopressors and pulmonary vasodilators whenever necessary as essential elements of management. ${ }^{(26)}$

One limitation of our study was the small sample size; only 40 patients could be enrolled during the study period. This restriction affected the multivariate risk regression analysis in that its revealed risk of IDH factors was unclear. Although female patients and patients with PHT showed a higher chance of IDH occurrence than their counterparts, the difference was not statistically significant.

\section{Conclusion}

Patients with ESRD and PHT measured by RVSP or mean PAP during echocardiography showed a higher tendency of developing IDH during hemodialysis than patients with ESRD without PHT. Therefore, patients with ESRD who were diagnosed having PHT showed clinical significance so that physicians had to closely monitor the possible occurrence of hypotension during hemodialysis.

\section{Conflict of interest}

The authors declare they have no conflict of interest.

\section{References}

1. Henrich WL. Hemodynamic instability during hemodialysis. Kidney Int 1986; 30: 605-12.

2. Zucchelli P, Santoro A. Dialysis-induced hypotension: a fresh look at pathophysiology. Blood Purif 1993; 11: 85-98.

3. Tisler A, Akocsi K, Harshegyi I, Varga G, Ferenczi S, Grosz M, et al. Comparison of dialysis and clinical characteristics of patients with frequent and occasional hemodialysisassociated hypotension. Kidney Blood Press Res 2002; 25: 97-102.

4. Shoji T, Tsubakihara Y, Fujii M, Imai E. Hemodialysis-associated hypotension as an independent risk factor for two-year mortality in hemodialysis patients. Kidney Inter 2004; 66: 1212-20.

5. Flythe JE, Xue H, Lynch KE, Curhan GC, Brunelli SM. Association of mortality risk with various definitions of intradialytic hypotension. J Am Soc Nephrol 2015; 26: 724-34.

6. Burton JO, Jefferies HJ, Selby NM, McIntyre CW. Hemodialysis-induced cardiac injury: determinants and associated outcomes. Clin J Am Soc Nephrol 2009; 4: 914-20.

7. Tarrass F, Benjelloun M, Medkouri G, Hachim K, Benghanem MG, Ramdani B. Doppler echocardiograph evaluation of pulmonary hypertension in patients undergoing hemodialysis. Hemodial Inter 2006; 10: 356-9.

8. Yigla M, Abassi Z, Reisner S, Nakhoul F.Pulmonary hypertension in hemodialysis patients: an unrecognized threat. Semin Dial 2006; 9: 353-57.

9. Li Z, Liu S, Liang X, Wang W, Fei H, $\mathrm{Hu}$, et al. Pulmonary hypertension as an independent predictor of cardiovascular mortality and events in hemodialysis patients. Inter Urol Nephrol 2014; 46: 141-9.

10. Fabbian F, Cantelli S, Molino C, Pala M, Longhini C, Portaluppi F. Pulmonary hypertension in dialysis patients: a cross-sectional Italian study. Int J Nephrol 2010; 2011: 283475. 
11. Ramasubbu K, Deswal A, Herdejurgen C, Aguilar D, Frost AE. A prospective echocardiographic evaluation of pulmonary hyper tension in chronic hemodialysis patients in the United States: prevalence and clinical significance. Int J Gen Med 2010; 3: 279-86.

12. Yigla M, Fruchter O, Aharonson D, Yanay N, Reisner SA, Lewin M, et al. Pulmonary hypertension is an independent predictor of mortality in hemodialysis patients. Kidney Inter 2009; 75: 969-75.

13. Agarwal R. Prevalence, determinants and prognosis of pulmonary hypertension among hemodialysis patients. Nephrol Dial Transplant 2012; 27: 3908-14.

14. K/DOQI clinical practice guidelines for cardiovascular disease in dialysis patients. Am J Kidney Dis $2005 ; 45$

15. Poldermans D, Man in 't Veld AJ, Rambaldi R, Van Den Meiracker AH, Van Den Dorpel MA, Rocchi G, et al. Cardiac evaluation in hypotension-prone and hypotension-resistant hemodialysis patients. Kidney Int 1999; 56: 1905-11.

16. Kanbay M, Ertuglu LA, Afsar B, Ozdogan E, Siriopol D, Covic A, et al. An update review of intradialytic hypotension:concept, risk factors, clinical implications and management. Clin Kidney J 2020; 13: 981-93.

17. Patrick B. Reeves, Finnian R. Mc Causland. Mechanisms, Clinical Implications, and Treatment of Intradialytic Hypotension. Clin J Am Soc Nephrol 2018; 13: 1297-303.

18. Kawar B, Ellam T, Jackson C, Kiely DG. Pulmonary hypertension in renal disease: epidemiology, potential mechanisms and implications. Am J Nephrol 2013; 37: 281-90.

19. Tisler A, Akocsi K, Harshegyi I, Varga G, Ferenczi S, Grosz M, et al. Comparison of dialysis and clinical characteristics of patients with frequent and occasional hemodialysis associated hypotension. Kidney Blood Press Res 2002; 25: 97-102.

20. Kuipers J, Verboom LM, Ipema KJR, Paans W, Krijnen WP, Gaillard CAJM, et al. The prevalence of intradialytic hypotension in patients on conventional hemodialysis: A systematic review with meta-analysis. Am J Nephrol 2019; 49: 497-506.

21. Kuipers J, Oosterhuis JK, Krijnen WP, Dasselaar JJ, Gaillard CAJM, Westerhuis R, et al. Prevalence of intradialytic hypotension, clinical symptoms and nursing interventionsa three-months, prospective study of 3818 haemodialysis sessions. BMC Nephrol 2016; 17: 1-11.

22. Orofino L, Marcén R, Quereda C, Villafruela JJ, Sabater J, Matesanz R, et al. Epidemiology of symptomatic hypotension in hemodialysis is cool dialysate beneficial for all patients? Am J Nephrol 1990; 10: 177-80.

23. Chao CT, Huang JW, Yen CJ. Intradialytic hypotension and cardiac remodeling: a vicious cycle. Biomed Res Int 2015: 724147.

24. Barberato SH, Misocami M, Pecoits-Filho R. Association between left atrium enlargement and intradialytic hypotension: role of diastolic dysfunction in the hemodynamic complications during hemodialysis. Echocardiography 2009; 26: 767-71.

25. Gille J, Seyfarth HJ, Gerlach S, Malcharek M, Czeslick E, Sablotzki A. Perioperative anesthesiological management of patients with pulmonary hypertension. Anesthesiol Res Pract 2012; 2012: 356982.

26. Minai OA, Yared JP, Kaw R, Subramaniam K, Hill NS. Perioperative risk and management in patients with pulmonary hypertension. Chest 2013; 144: 329-40. 